2009, 113, 8210-8213

Published on Web 05/22/2009

\title{
Does the Dynamic Stokes Shift Report on Slow Protein Hydration Dynamics?
}

\author{
Bertil Halle $*, \dagger^{*}$ and Lennart Nilsson $*, \sharp$ \\ Biophysical Chemistry, Center for Molecular Protein Science, Lund University, SE-22100 Lund, Sweden, and \\ Department of Biosciences and Nutrition, Karolinska Institutet, SE-14157 Huddinge, Sweden
}

Received: March 26, 2009; Revised Manuscript Received: May 12, 2009

\begin{abstract}
The time-dependent fluorescence frequency shift of protein-attached probes has a much slower decay than that for the free probe. The decay times, ranging from $10 \mathrm{ps}$ to several nanoseconds, have been attributed to hydration water motions several orders of magnitude slower than those in the hydration shell of small solutes. This interpretation deviates strongly from the prevailing picture of protein hydration dynamics. We argue here that the slow decay in the fluorescence shift can be explained by a ubiquitous solvent polarization mechanism, with no need to invoke slow water motions or a dynamic coupling with protein motions. This mechanism can be qualitatively understood with the aid of a dielectric continuum model. We therefore conclude that the long decay times measured with time-dependent fluorescence spectroscopy contain no information about protein hydration dynamics.
\end{abstract}

Many biological processes depend critically on the dynamical properties of the water-protein interface. During the past half century, a diverse array of techniques has therefore been recruited to the challenging task of quantitatively characterizing, under physiological solution conditions, the motions of water molecules in the protein hydration layer. ${ }^{1}$ The current picture, founded largely on ${ }^{17} \mathrm{O}$ NMR relaxation ${ }^{2-5}$ and MD simulation ${ }^{6-11}$ studies, reveals a dynamically heterogeneous hydration layer; a few water molecules spend long periods (up to $\sim 1 \mathrm{~ns}$ ) trapped in surface pockets, while $\sim 90 \%$ of the interfacial water molecules, like those in the hydration shell of small organic solutes, suffer a mere two-fold average dynamical retardation as compared to bulk water. In recent years, this picture has been challenged by time-resolved fluorescence measurements, which seem to indicate that water motions in the hydration layer are slowed down by several orders of magnitude. ${ }^{12-22}$ In this Letter, we argue that the long decay times seen by this technique reflect protein conformational fluctuations rather than hydration dynamics. There is thus no need to revise the prevailing picture of protein hydration dynamics.

The fluorescence-detected dynamic Stokes shift (DSS) monitors the interaction energy of a fluorescent probe with its molecular environment. The charge distribution of the probe is altered by electronic excitation, and the ensuing readjustment of the environment is reflected in the time-dependent DSS. The DSS from a free probe in aqueous solution exhibits a sub-100 fs inertial decay of large amplitude, followed by a diffusive decay with a characteristic time of $\sim 1 \mathrm{ps}^{23,24}$ In a dielectric model, the longer decay time corresponds to the dipolar longitudinal relaxation time of the solvent, which is $0.6 \mathrm{ps}$ for bulk water at $298 \mathrm{~K}$.

* To whom correspondence should be addressed. Phone: +46 462229516 (B.H.); +46 86089228 (L.N.). E-mail: bertil.halle@bpc.lu.se (B.H.); lennart.nilsson@ki.se (L.N.).

${ }^{\dagger}$ Lund University.

* Karolinska Institutet.
When the probe is attached to a protein, the DSS exhibits one or more slowly decaying components in addition to the inertial decay (which is not always resolved) and a fast diffusive decay of a few picoseconds (corresponding to the $\sim 1 \mathrm{ps}$ component of the free probe). The molecular origin of the longest decay time, which may be anywhere from $10 \mathrm{ps}$ to several nanoseconds, is not fully understood. Zewail and coworkers argue that these long decay times characterize water motions in the protein hydration layer, either exchange between hydration and bulk water ${ }^{12-17,19}$ or more subtle local rearrangements of the orientation and density of hydration water. ${ }^{20,22}$ However, on the basis of ${ }^{17} \mathrm{O} \mathrm{NMR}^{2-5}$ and MD simulation ${ }^{6-11}$ results, we expect such water motions to occur on a time scale of a few picoseconds, corresponding to the faster observed DSS decay. The few relatively long-lived hydration water molecules undergo restricted motions on a similar (picosecond) time scale, and their much slower exchange with bulk water does not, for symmetry reasons, affect the DSS (see below). Then, what is the origin of the slow DSS decay?

To fully answer this question, we must link the slow DSS decay to a molecular component (protein or water) and to a specific type of motion. The required dissection of the DSS can be accomplished by MD simulations. ${ }^{25-32}$ To a good approximation, the difference in probe solvation energy between the excited and ground states, $\Delta E$, can be modeled as a sum of Coulomb interactions. The DSS can then be uniquely decomposed into protein and water contributions, $S(t)=S^{\mathrm{P}}(t)+S^{\mathrm{W}}(t)$. For the single tryptophan residue in the protein monellin ${ }^{25}$ and for the drug molecule $\mathrm{H} 33258$ bound to DNA, ${ }^{30} \mathrm{MD}$ simulations yield a decay time of $\sim 1.5 \mathrm{ps}$ for the water component $S^{\mathrm{W}}(t)$, and the slow DSS component is found to be associated with the biomolecule rather than, as initially assumed, ${ }^{13,33}$ with the water.

Further insights into the molecular origin of the slow DSS component have come from MD simulations showing that the relative importance of protein and water depends on the structure 
(a)
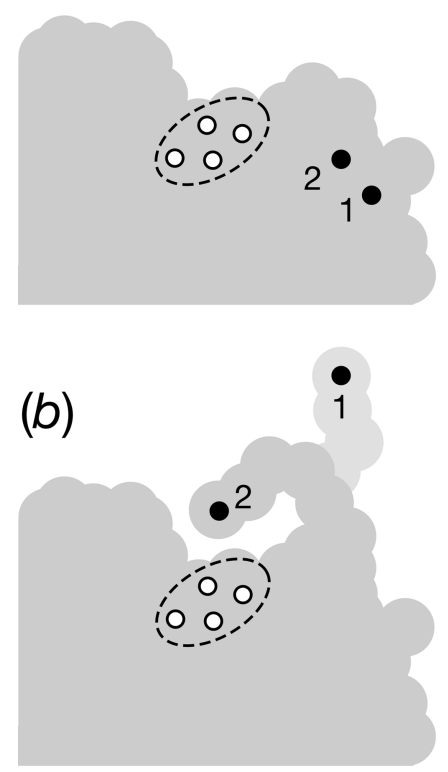

Figure 1. Schematic view of a probe (ellipse) near the surface of a protein (gray). The solid circles labeled 1 and 2 represent alternative locations of a fluctuating protein atom bearing a partial charge. The open circles represent partial charges mimicking the excited-to-groundstate differential charge distribution of the probe.

and flexibility of the protein in the vicinity of the probe. ${ }^{29,31}$ Of particular interest is the observation by Singer et al. that the slowly decaying water contribution $S^{\mathrm{W}}(t)$, if observable, disappears when the protein conformation is frozen. ${ }^{31}$ This finding, and their analysis of water motions in the hydration layer, led these authors to propose that the slow water component in the DSS arises from water translational motion coupled to protein motions. ${ }^{31}$ The compensating and coupled variations in the protein and water contributions to the solvation energy documented by Singer et al. had actually been anticipated on the basis of a simple dielectric model. ${ }^{25}$ In the following, we use this model to argue more explicitly that the mechanism of the slow water contribution differs fundamentally from that proposed by Singer et al. and that, as a consequence, the slow DSS decay contains no information at all about protein hydration dynamics.

In our highly simplified model (Figure 1a), the protein can exist in either of two conformations, which differ in the position (1 or 2) of an atom bearing a partial charge (for example, as prescribed by a molecular mechanics force field). The energy difference $\Delta E$ is then given by the interaction of the probe with the single protein charge and with all surrounding water molecules. The protein relaxation time $\tau_{\mathrm{P}}$ is related to the lifetimes of the two states $\tau_{\mathrm{P}}=\left(1 / \tau_{1}+1 / \tau_{2}\right)^{-1}$. The water is modeled as a dielectric continuum with relative permittivity $\varepsilon$. To describe nonequilibrium hydration at short times, we could use a frequency-dependent permittivity, ${ }^{34}$ but the static permittivity suffices since we are only interested in the DSS decay on time scales $\tau_{\mathrm{P}}$ much longer than the longitudinal relaxation time $\tau_{\mathrm{W}}$ of the (hydration) water. At the cost of numerical complexity, we could take the dielectric boundary into account, ${ }^{35,36}$ but such details are unimportant here; therefore, we regard $\varepsilon$ as the effective permittivity of the local interfacial region.

For this model, the excited-to-ground-state differential solvation energy of the probe is simply $\Delta E=\Delta E^{\mathrm{P}} / \varepsilon$, where $\Delta E^{\mathrm{P}}$ is the bare interaction energy (without water). Consequently, $\Delta E^{\mathrm{W}} \equiv \Delta E-\Delta E^{\mathrm{P}}=-(1-1 / \varepsilon) \Delta E^{\mathrm{P}}$. Since the water is in equilibrium with the protein conformation on the time scale $\tau_{\mathrm{P}}$, the water and protein contributions to the DSS are related in the same way as the interaction energy

$$
S^{\mathrm{W}}(t)=-(1-1 / \varepsilon) S^{\mathrm{P}}(t)
$$

This simple result tells us that even if the water molecules move at infinite rate $\left(\tau_{\mathrm{W}}=0\right)$, the DSS contains a water contribution that decays on the long time scale $\tau_{\mathrm{P}}$ of protein relaxation. The water and protein contributions are of opposite sign, and if $\varepsilon \gg$ 1 (as expected if the probe and charge are near the protein surface), they are of similar magnitude; therefore, the total DSS is much smaller than either component. Despite the simplicity of the model, eq 1 agrees qualitatively with the slowly decaying DSS components in apomyoglobin. ${ }^{31}$ Furthermore, the model reproduces the observation ${ }^{31}$ that the slow water contribution disappears when the protein conformation is frozen, that is, when $S^{\mathrm{P}}(t) \equiv 0$ in eq 1 . The model can be trivially extended to an arbitrary number of partial charges and conformational states, as in a real protein. Although the total $S(t)$ must decrease with time, the $S^{\mathrm{P}}(t)$ contributions from the individual charges may increase or decrease because the protein atoms do not, in general, move independently of each other. One can therefore envisage situations with mutual cancellation among the various protein contributions. According to the model, each protein contribution is accompanied by a water contribution of opposite sign. However, the effective permittivity $\varepsilon$ depends on the location of the charge; therefore, the $S^{\mathrm{W}}(t)$ contributions may cancel each other more or less completely than the $S^{\mathrm{P}}(t)$ contributions do. Such cancellation of individual charge contributions was observed in the apomyoglobin simulation. ${ }^{31}$

Some MD studies ${ }^{26-28,32}$ have decomposed the DSS as $S(t)$ $=S^{\mathrm{PP}}(t)+S^{\mathrm{WW}}(t)+S^{\mathrm{PW}}(t)$, although these terms cannot be identified with the protein and water contributions to the measured DSS. ${ }^{25,37}$ For this decomposition scheme, our model predicts that

$$
S^{\mathrm{WW}}(t)=(1-1 / \varepsilon)^{2} S^{\mathrm{PP}}(t)=-(1-1 / \varepsilon) S^{\mathrm{PW}}(t) / 2
$$

For $\varepsilon \gg 1$, the water-water and protein-protein contributions are equal in magnitude and sign, while the protein-water cross term is of opposite sign and 2-fold larger in magnitude. Again, the model agrees remarkably well with the simulation results (where the cross term is displayed as $\left.S^{\mathrm{PW}}(t) / 2\right) .^{26,27}$

Despite its simplicity, the dielectric model can reproduce, at least qualitatively, the range of DSS behaviors revealed by MD simulations. According to this model, the slow water contribution to the DSS arises from the polarization of the solvent induced by the time-dependent electric field from the probe and protein charges. Previously, the long decay time of this contribution has been linked to water motions in the hydration layer. ${ }^{12-22,26,27,31-33}$ However, the polarization of the solvent is produced by very slight orientational preferences among a large number of water molecules, within and beyond the hydration layer. More importantly, the polarization is established on a time scale $\tau_{\mathrm{W}}$ (one or a few picoseconds) much shorter than the time scale $\tau_{\mathrm{P}}$ on which $S^{\mathrm{W}}(t)$ decays. Therefore, the wide range of slow decay times obtained with tryptophan probes in proteins, 10-200 ps, reflects the multiple time scales of internal protein dynamics rather than differences in hydration dynamics. If the DSS could be recorded in a wider time window, even longer decay times would be obtained, produced by protein confor- 
mational dynamics on nanosecond, microsecond, and millisecond time scales and with the associated water polarization contributions. Indeed, using extrinsic probes with more longlived excited states, DSS decays of several nanoseconds have been reported. ${ }^{38-42}$ According to our analysis, the decay time of the slow $S^{\mathrm{W}}(t)$ component does not contain any information about protein hydration dynamics. It is therefore misleading to state that "we have determined the actual time scale of coupled water-protein fluctutations". ${ }^{21}$ Such a time scale does not exist; $S^{\mathrm{W}}(t)$ can decay on any and all time scales on which protein internal motions occur. A close analogy exists with dielectric relaxation spectroscopy. The electric polarization of a bulk water sample follows the oscillations of an applied AC field at any frequency below $1 / \tau_{\mathrm{D}} \approx 0.1 \mathrm{THz}$. However, measurements below this frequency tell us nothing about water dynamics.

The water-protein coupling underlying the slowly decaying $S^{\mathrm{W}}(t)$ component is not a dynamic coupling. It is therefore misleading to attribute the slow $S^{\mathrm{W}}(t)$ decay to coupled water-protein fluctuations. ${ }^{21,27,31}$ If protein and water motions occur on widely disparate time scales, as assumed in our model $\left(\tau_{\mathrm{W}} \ll \tau_{\mathrm{P}}\right)$, then they are necessarily statistically independent and cannot exhibit a dynamic coupling. The mechanism behind the slowly decaying $S^{\mathrm{W}}(t)$ component has also been described as "coupled water-protein translation". ${ }^{31}$ However, as our model shows, the slow $S^{\mathrm{W}}(t)$ decay occurs even if the protein-water boundary is unaffected by the internal motion (Figure 1a). Furthermore, when water displacement is involved, as in the slow reorientation of an exposed side chain (Figure 1b), it may affect $S^{\mathrm{W}}(t)$ by altering the effective permittivity $\varepsilon$ in eq $1,{ }^{29}$ but it does not influence the decay time as long as $\tau_{\mathrm{W}} \ll \tau_{\mathrm{P}}$.

The hydration layer of a protein is dynamically heterogeneous, with a power law distribution of residence times and rotational correlation times in the picosecond to nanosecond range (at room temperature). ${ }^{4,8,10}$ If one of the few "slow" water molecules happened to reside near the probe, how would the DSS be affected? To answer this question, we return to our model (Figure 1a). The positional fluctuation of the protein charge contributes to the DSS because the modified charge distribution of the newly excited probe slightly alters the relative populations of the two states 1 and 2. When a long-lived (>several $100 \mathrm{ps)}$ water molecule leaves its hydration site on the protein surface, it is immediately (within a few picoseconds) replaced by another water molecule from the bulk. Since the final state of this water exchange process is identical to the initial state, there can be no excitation-induced population change. Such slow water exchange processes are dynamically coupled to (local) conformational fluctuations in the protein, but they are invisible to the DSS technique.

In conclusion, for the reasons given above, our answer to the title question is no. A slowly decaying water contribution $S^{\mathrm{W}}(t)$ in the DSS is a necessary but not a sufficient condition for inferring slow hydration water dynamics. Such a contribution is always present, whether observable or not, and it would be there even if hydration water behaved exactly as bulk water. The slowly decaying DSS component observed with proteins but not with small organic solutes (such as the probe molecule itself) does not indicate a difference in hydration dynamics but results from the slow conformational fluctuations that occur in proteins but not in small solutes. Since all parts of a protein are more or less polar (as illustrated by the partial charges carried by all protein atoms in molecular mechanics force fields), the solvent polarization mechanism, proposed here as the source of the slow DSS decay in protein solutions, is ubiquitous. Any protein motion, on any time scale longer than $\sim 1 \mathrm{ps}$, is accompanied by a water component in the DSS that decays on the same time scale as the protein motion. However, this contribution does not report on the time scale of water molecule motions. This general conclusion does not rely on the quantitative approximations inherent in the dielectric continuum model used here mainly as a heuristic tool to illustrate the underlying physical mechanism.

Acknowledgment. This work was supported by the Swedish Research Council.

\section{References and Notes}

(1) Kuntz, I. D.; Kauzmann, W. Adv. Protein Sci. 1974, 28, 239-345.

(2) Halle, B. Water in biological systems: The NMR picture. In Hydration Processes in Biology: Theoretical and Experimental Approaches; Bellissent-Funel, M. C., Ed.; IOS Press: Dordrecht, 1999; pp 233-249. 1224.

(3) Halle, B. Philos. Trans. R. Soc. London, Ser. B 2004, 359, 1207-

(4) Mattea, C.; Qvist, J.; Halle, B. Biophys. J. 2008, 95, 2951-2963.

(5) Qvist, J.; Persson, E.; Mattea, C.; Halle, B. Faraday Discuss. 2009, $141,131-144$.

(6) Makarov, V. A.; Andrews, B. K.; Smith, P. E.; Pettitt, B. M. Biophys. J. 2000, 79, 2966-2974.

(7) Luise, A.; Falconi, M.; Desideri, A. Proteins 2000, 39, 56-67.

(8) García, A. E.; Hummer, G. Proteins 2000, 38, 261-272.

(9) Marchi, M.; Sterpone, F.; Ceccarelli, M. J. Am. Chem. Soc. 2002, 124, 6787-6791.

(10) Henchman, R. H.; McCammon, J. A. Protein Sci. 2002, 11, 20802090 .

(11) Bizzarri, A. R.; Cannistraro, S. J. Phys. Chem. B 2002, 106, 66176633

(12) Pal, S. K.; Peon, J.; Zewail, A. H. Proc. Natl. Acad. Sci. U.S.A. 2002, 99, 1763-1768.

(13) Peon, J.; Pal, S. K.; Zewail, A. H. Proc. Natl. Acad. Sci. U.S.A. 2002, 99, 10964-10969.

(14) Pal, S. K.; Peon, J.; Zewail, A. H. Proc. Natl. Acad. Sci. U.S.A. 2002, 99, 15297-15302.

(15) Pal, S. K.; Peon, J.; Bagchi, B.; Zewail, A. H. J. Phys. Chem. B 2002, 106, 12376-12395.

(16) Bhattacharyya, S. M.; Wang, Z.-G.; Zewail, A. H. J. Phys. Chem. B 2003, 107, 13218-13228.

(17) Kamal, J. K. A.; Zhao, L.; Zewail, A. H. Proc. Natl. Acad. Sci. U.S.A. 2004, 101, 13411-13416.

(18) Zhao, L.; Pal, S. K.; Xia, T.; Zewail, A. H. Angew. Chem., Int. Ed. 2004, 43, 60-63.

(19) Pal, S. K.; Zewail, A. H. Chem. Rev. 2004, 104, 2099-2123.

(20) Qiu, W.; Kao, Y.-T.; Zhang, L.; Yang, Y.; Wang, L.; Stites, W. E.; Zhong, D.; Zewail, A. H. Proc. Natl. Acad. Sci. U.S.A. 2006, 103, 1397913984.

(21) Li, T.; Hassanali, A. A.; Kao, Y.-T.; Zhong, D.; Singer, S. J. J. Am. Chem. Soc. 2007, 129, 3376-3382.

(22) Zhang, L.; Wang, L.; Kao, Y.-T.; Qiu, W.; Yang, Y.; Okobiah, O.; Zhong, D. Proc. Natl. Acad. Sci. U.S.A. 2007, 104, 18461-18466.

(23) Jiminez, R.; Fleming, G. R.; Kumar, P. V.; Maroncelli, M. Nature 1994, 369, 471-473.

(24) Shen, X.; Knutson, J. R. J. Phys. Chem. B 2001, 105, 6260-6265. (25) Nilsson, L.; Halle, B. Proc. Natl. Acad. Sci. U.S.A. 2005, 102, 13867-13872.

(26) Bandyopadhyay, S.; Chakraborty, S.; Balasubraminian, S.; Bagchi, B. J. Am. Chem. Soc. 2005, 127, 4071-4075.

(27) Bandyopadhyay, S.; Chakraborty, S.; Bagchi, B. J. Phys. Chem. B 2006, 110, 20629-20634.

(28) Pal, S.; Maiti, P. K.; Bagchi, B.; Hynes, J. T. J. Phys. Chem. B 2006, 110, 26396-26402.

(29) Gosolov, A. A.; Karplus, M. J. Phys. Chem. B 2007, 111, 14821490 .

(30) Furse, K. E.; Corcelli, S. A. J. Am. Chem. Soc. 2008, 130, 1310313109.

(31) Li, T.; Hassanali, A. A.; Singer, S. J. J. Phys. Chem. B 2008, 112, 16121-16134.

(32) Sen, S.; Andreatta, D.; Ponomarev, S. Y.; Beveridge, D. L.; Berg, M. A. J. Am. Chem. Soc. 2009, 131, 1724-1735.

(33) Pal, S. K.; Zhao, L.; Zewail, A. H. Proc. Natl. Acad. Sci. U.S.A. 2003, 100, 8113-8118.

(34) Hsu, C.-P.; Song, X.; Marcus, R. A. J. Phys. Chem. B 1997, 101, 2546-2551.

(35) Song, X.; Chandler, D. J. Chem. Phys. 1998, 108, 2594-2600.

(36) Jordanides, X. J.; Lang, M. J.; Song, X.; Fleming, G. R. J. Phys. Chem. B 1999, 103, 7995-8005. 
(37) Bernard, W.; Callen, H. B. Rev. Mod. Phys. 1959, 31, 1017-1044.

(38) Pal, S. K.; Mandal, D.; Sukul, D.; Sen, S.; Bhattacharyya, K. J. Phys. Chem. B 2001, 105, 1438-1441.

(39) Sen, P.; Mukherjee, S.; Dutta, P.; Halder, A.; Mandal, D.; Banerjee, R.; Roy, S.; Bhattacharyya, K. J. Phys. Chem. B 2003, 107, 14563-14568. (40) Guha, S.; Sahu, K.; Roy, D.; Mondal, S. K.; Roy, S.; Bhattacharyya, K. Biochemistry 2005, 44, 8940-8947.
(41) Samaddar, S.; Mandal, A. K.; Mondal, S. K.; Sahu, K.; Bhattacharyya, K.; Roy, S. J. Phys. Chem. B 2006, 110, 21210-21215.

(42) Abbyad, P.; Shi, X.; Childs, W.; McAnaney, T. B.; Cohen, B. E.; Boxer, S. G. J. Phys. Chem. B 2007, 111, 8269-8276.

JP9027589 